\title{
CANDIDOSES : BASES MOLÉCULAIRES DE L'ADAPTATION PARASITAIRE DE PROTISTES PATHOGÈNES OPPORTUNISTES
}

\author{
D. POULAIN
}

\begin{abstract}
RÉSUMÉ
La levure endosaprophyte $C$. albicans exprime de manière facultative toutes les composantes de la pathogénicité retrouvées chez les protozoaires parasites obligatoires. En relation avec les particularités structurales de ce micro-organisme, les connaissances actuelles sur les bases moléculaires de l'adhérence, de la pénétration, de l'interaction avec les facteurs spécifiques et non spécifiques de l'immunité sont brièvement exposées. Les originalités parasitologiques majeures du modèle sont relatives à l'importance
\end{abstract}

prépondérante des variations phénotypiques. Celles-ci affectent particulièrement la paroi qui conditionne chacune des étapes liées aux processus invasifs, notamment par l'intermédiaire des mannoprotéines. Les Candida, qui expriment sur ces molécules un répertoire oligomannosidique extrêmement varié fournissent un modèle privilégié pour l'étude des relations entre ces structures, communes à de nombreux parasites, et leurs interactions avec l'hôte.

\section{Summary: Candidosis: molecular basis of parasitic adaptation of opportunistic fungal pathogens.}

Candida albicans is a versatile organism living as a commensal of the gastro-intestinal tract and having the ability to invade host tissues and to initiate serious diseases under the appropriate environmental conditions. The molecular basis for adherence, invasion, interactions with specific and non-specific immune factors have been studied in parallel to structural characteristics of the yeast. The main parasitologic features are closely linked to phenotypic variations. In this respect, mannoproteins are strongly involved in the cell wall variations. The study of the oligomannosidic repertoire represents one of the essential steps for the understanding of host-parasite relationships.

\section{INTRODUCTION}

La fin de ce siècle a vu l'avènement d'une nouvelle pathologie, tout d'abord restreinte aux pays à haut niveau de vie, la pathologie opportuniste. Les modifications écoéthologiques, l'évolution des techniques médico-chirurgicales, l'infection par le VIH ont conduit à l'expression du pouvoir pathogène d'organismes saprophytes pour lesquels le parasitisme n'est pas une « condition de vie normale et nécessaire ». La recrudescence de la pathologie fongique ces dernières années montre que c'est au sein de l'embranchement des champignons que se trouvaient les microorganismes potentiellement les plus adaptés à un tel comportement.

La mycologie médicale est en France une discipline associée à la parasitologie. Il paraît dès lors intéressant, dans le cadre d'une rencontre internationale sur les zooparasites, d'exposer et de discuter un certain nombre d'acquis récents sur les bases moléculaires de l'adaptation parasitaire de champignons opportunistes. L'espèce $C$. albicans, commen-

Service de Parasitologie-Mycologie. Faculté de Médecine, Place Verdun, F 59000 Lille et Unité INSERM 42, F 59651 Villeneuve d'Ascq Cedex. (Directeur : Pr D. Camus). sale habituelle du tube digestif humain, responsable d'infections qui sont par leur fréquence et leur gravité au premier rang des infections opportunistes, est à cet égard un exemple privilégié.

I - Organisation générale de la cellule de C. albicans

C. albicans est une levure polymorphique, susceptible de croître sous forme filamenteuse (10). Quelle que soit la morphologie cellulaire, l'organisation ultrastructurale du cytoplasme ne présente pas de différenciation fondamentale par rapport à l'organisation générale des cellules eucaryotes, une partie importante du métabolisme concerne l'édification et les remaniements de la paroi qui représente jusqu'à $15 \%$ du poids sec de la cellule (fig. 1). Celle-ci s'organise à partir de l'espace périplasmique situé immédiatement à l'extérieur du plasmalemme. Elle est constituée (fig. $l$ b) d'un réseau très dense de fibrilles insolubles de glucanes (polymères de $\beta 1-3$ et de $\beta 1-6$ glucose) associées à de la chitine (polymère de $\beta 1-4$ glucosamine acétylée). Dans les mailles du réseau ainsi formé sont logés des composés dits matriciels, constitués pour l'essentiel de mannoprotéines complexes (fig. 2) qui sont extractibles par des solvants aqueux. Les mannoprotéines sont soit structurales 


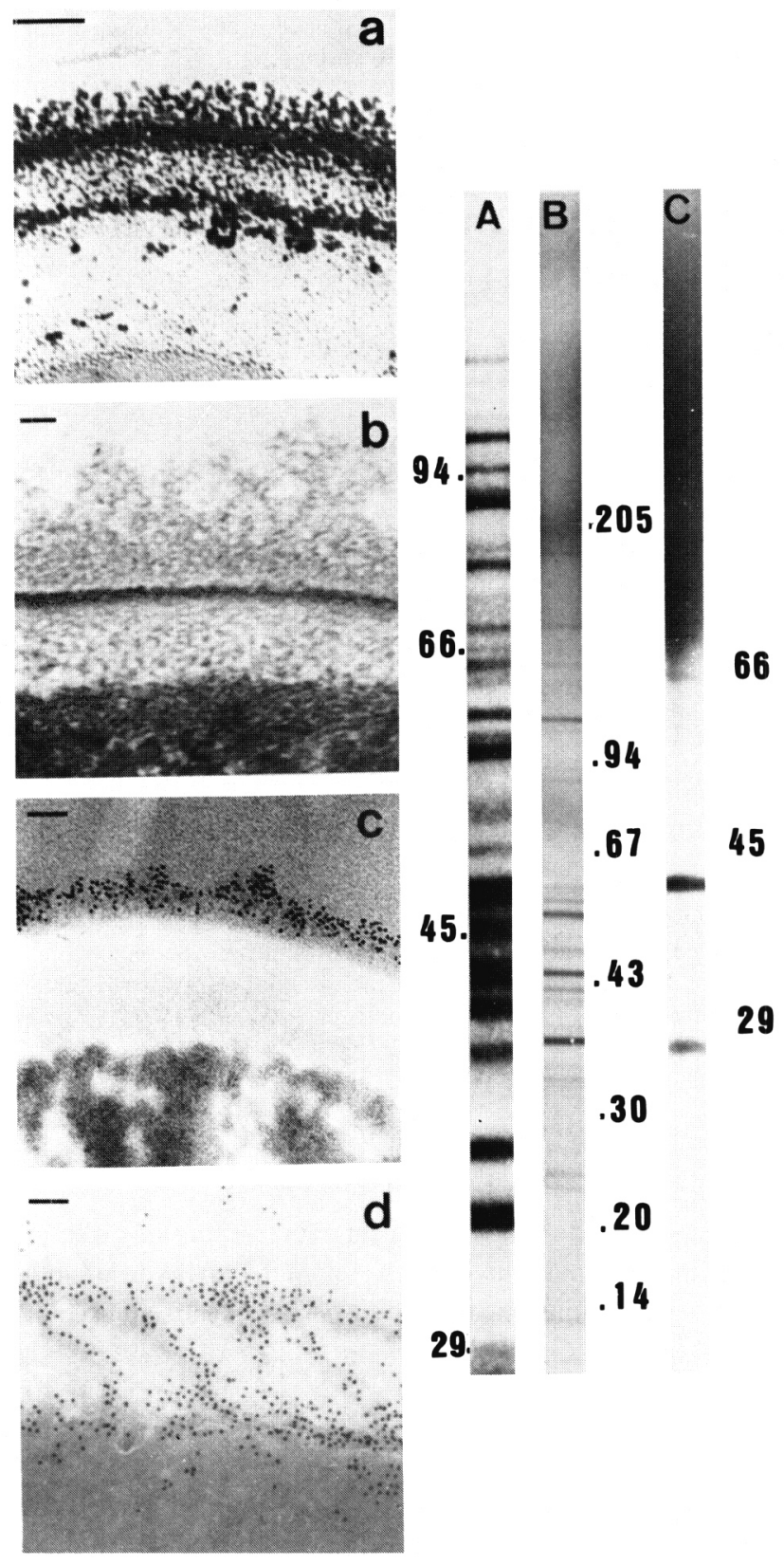

FIG. 1. - $a-c$ : organisation de la paroi objectivée par différentes méthodes de détection ultrastructurale. $a$ ) distribution des mannoprotéines - denses aux électrons - à la surface cellulaire et dans l'espace périplasmique; $b$ ) réseau de fibrilles responsable de la rigidité pariétale. Cellules incubées dans du sérum humain normal, remarquer la couche épaisse de protéines sériques adsorbées à la surface pariétale; $c$ ) marquage immunologique de surface révélant la présence d'une zone translucide le manteau muqueux - qui recouvre les structures pariétales plus denses; $d$ ) voies de cheminement préférentielles de mannoprotéines excrétées dans le milieu de culture, révélées au sein de la paroi par immunodétection impliquant un anticorps monoclonal. La barre portée sur les micrographies représente $0,1 \mu \mathrm{m}$. A-C : autoradiographies de différents profils électrophorétiques en gel de polyacrylamide après marquage métabolique à la méthionine. A) protéines cytoplasmiques. B) extrait pariétal obtenu après action de glucanase. C) produits d'excrétion-secrétion spontanément relargués dans un milieu de culture synthétique. et contribuent à renforcer la rigidité pariétale, soit enzymatiques et interviennent dans le métabolisme de la paroi ainsi que dans les nombreux échanges se produisant entre la cellule et le milieu extérieur. La surface pariétale paraît le plus fréquemment recouverte de fibrilles de faible diamètre baignant dans un manteau muqueux (fig. $l \mathrm{a}, l \mathrm{~b}$ ). Il existe enfin, au sein de la paroi, des différenciations radiaires correspondant aux zones de passage préférentielles de composants mis en place à la surface cellulaire ou excrétés dans le milieu (fig. 1 c).

Le marquage métabolique de cellules de $C$. albicans par la méthionine révèle, après électrophorèse en gel de polyacrylamide, un profil relativement classique des protéines cytoplasmiques (fig. $1 \mathrm{~A}$ ). L'électrophorèse des protéines libérées de la paroi par digestion du réseau de glucanes révèle l'existence de glycoprotéines de haut poids moléculaire à forte copule polysaccharidique, se présentant sous forme de traînées dans le gel (fig. 1 B). L'analyse du milieu de culture révèle la même importance prépondérante des glycoprotéines au sein des produits d'excrétionsécrétion (fig. $1 \mathrm{C}$ ).

\section{II - L'expression du pouvoir pathogène}

L'initiation du mécanisme de pathogénicité est liée à l'existence de " causes favorisantes " nombreuses et variées dépendantes de l'hôte (10). Il s'agit : $i$ ) de facteurs locaux détruisant l'équilibre écologique de la peau comme les détergents, ou des muqueuses comme les antibiotiques antibactériens; ii) d'actes médico-chirurgicaux invasifs introduisant des levures dans un environnement normalement stérile; iii) d'immunodépressions transitoires ou prolongées, relatives à des pathologies variées, à l'administration de drogues immunosuppressives, ou à l'infection par des microorganismes eux-mêmes immunosuppresseurs. Ces différents facteurs peuvent se conjuguer chez un même patient, augmentant alors considérablement le risque d'infection candidosique.

\section{III - Les composantes multifactorielles de la pathogénicité}

Des causes multiples aboutissent donc à un phénomène unique, l'invasion tissulaire de l'hôte, circonscrite ou généralisée. D'un point de vue parasitologique, le déterminisme de cette pathogénicité est le fondement de nombreuses recherches sur la biologie de $C$. albicans visant à en identifier les mécanismes moléculaires.

\section{A - Colonisation et adhÉrence AuX MUQueUses}

L'adhérence de $C$. albicans aux muqueuses, mais également aux mucines qui tapissent le tractus digestif, est une condition nécessaire à la colonisation de son habitat naturel chez l'hôte et à l'infection susceptible d'en résulter. 


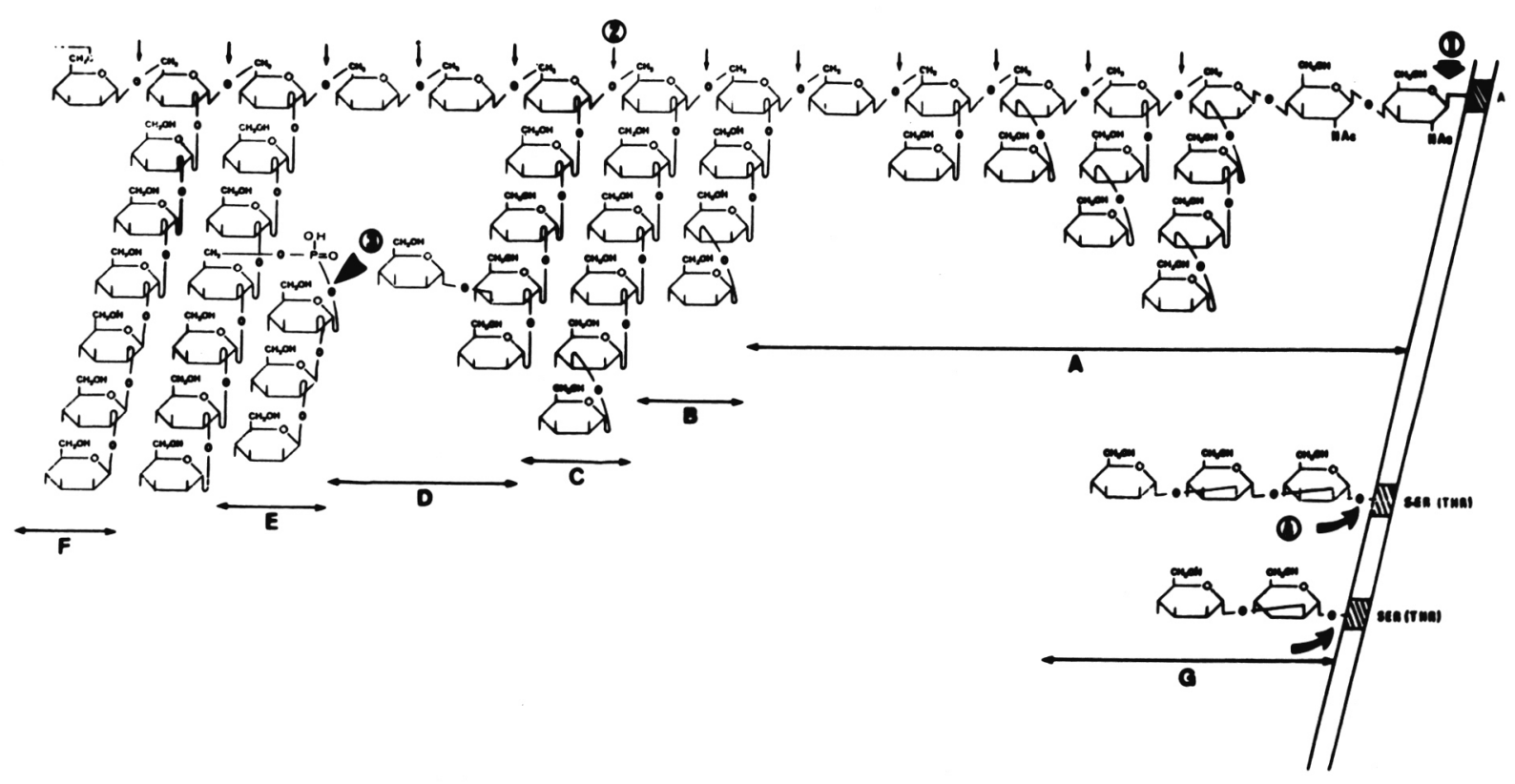

FIG. 2. - Schéma synthétique de la structure des phosphopeptidomannanes de la paroi de C. albicans. Des liaisons O-glycosidiques (G) relient aux protéines de courtes chaînes oligomannosidiques, les liaisons $\mathrm{N}$-glycosidiques concernent des édifices macromoléculaires plus complexes. La chaîne interne $(\mathrm{A})$ est en partie commune à tous les stades précoces de biosynthèse des glycoprotéines chez les cellules eucaryotes. Des chaînes latérales d'oligomannosides, linéaires ou ramifiées, comportant des liaisons $\alpha 1-2, \alpha 1-3$ et $\beta 1-2$ (B, C, D, E, F) sont branchées sur une longue chaîne linéaire formée de mannose lié en $\alpha 1-6$.

L'adhérence procède du déroulement séquentiel d'événements physico-chimiques au cours desquels interviennent la charge de surface des levures (négative), des interactions hydrophobes (les souches les plus hydrophobes sont les plus adhérentes), puis des interactions plus spécifiques impliquant de véritables ligands de la surface cellulaire définis comme des adhésines (4). Les résultats d'expériences d'inhibition de l'adhérence cellulaire par divers composés ont abouti à des résultats extrêmement variés, souvent contradictoires quant à la nature présumée des adhésines. Les expériences d'inhibition par les sucres aminés, le mannose, le traitement par enzymes protéolytiques ou les stérols ont conduit respectivement à suspecter l'implication de la chitine, de la copule polysaccharidique des mannoprotéines, de glycolipides. L'extrême variabilité de la surface pariétale dépendante des souches, liée au manque de standardisation des protocoles expérimentaux (milieu de culture, nature des cellules humaines), explique en partie ces discordances. Il est par contre clair que les formes germinatives adhèrent davantage que les blastoconidies matures à de nombreux substrats, y compris les substances acryliques. Ce dernier modèle a permis l'identification de quatre composants exprimés préférentiellement sur la paroi du tube germinatif intervenant dans l'adhérence. Il s'agit de glycoprotéines de poids moléculaires 60000,68000 ainsi que de deux composés à copule polysaccharidique plus importante dont l'un présente une masse moléculaire apparente de 200000 (15).

\section{B - PÉnÉtration tissulaire}

La pénétration tissulaire nécessite des équipements enzymatiques complexes, tout particulièrement lorsqu'elle s'opère dans des tissus comportant des scléroprotéines tels que l'ongle, les épithelia œsophagiens ou vaginaux. Parmi plus de 40 enzymes décrites chez C. albicans, les protéases, la lysophospholipase ont été décrites comme intervenant dans la lyse tissulaire. La simple germination d'une spore pénétrant les tissus nécessite une croissance orientée. La forme cellulaire étant déterminée par l'agencement des microfibrilles pariétales, la pénétration nécessite donc la mise en œuvre de phénomènes extrêmement complexes qui dirigent . l'édification macromoléculaire de la paroi néoformée. Malgré de très nombreux travaux sur la morphogénèse, en particulier sur la transformation levure-mycélium, aucune conclusion n'a pu être obtenue concernant l'influence prépondérante d'un facteur externe déclenchant. Seule, l'analyse mathématique permet de rendre compte du caractère multifactoriel des innombrables signaux susceptibles d'influer sur la cellule ( $\mathrm{pH}$, température, cAMP, proline, amino-acides, densité cellulaire...) (3). Plusieurs facteurs ont été identifiés à l'intérieur de la cellule en tant que seconds messagers potentiels de la transformation morphologique, il s'agit de l'AMP cyclique, de la calmoduline et du $\mathrm{pH}$ intracellulaire (5). L'analyse du contrôle génétique de ce phénomène, qui apparaît pour l'instant essentiellement phénotypique, est encore hors de portée. 


\section{C - DissÉminAtion, SURVIE ET PROLIFÉRATION DANS L'ORGANISME}

Les levures et le mycélium ayant pénétré l'organisme vont se trouver au contact de différents tissus aux dépens desquels ils vont croître, tout en luttant contre les mécanismes spécifiques et non spécifiques responsables du maintien de l'homéostasie de l'hôte. Nous envisagerons successivement les interactions complexes qui ont été démontrées dans chacun de ces domaines, et dont la résultante aboutira à la dissémination des Candida ou à la résistance à l'infection.

\section{- Interactions avec les composants tissulaires et humoraux non liés à l'immunité spécifique}

De nombreuses interactions ont été décrites entre la paroi de $C$. albicans et des composants humoraux et tissulaires. Il a ainsi été démontré que les mannanes pariétaux fixaient la fraction C3 du complément induisant l'activation de la voie alterne. La fibronectine, le fibrinogène, la laminine, la « C-reactive protein », la « mannose binding protein », le lysosyme, la transferrine, l'albumine, sont autant d'autres composés pour lesquels une fixation à la surface de C. albicans a été rapportée. La signification physiopathologique de l'ensemble de ces observations est d'interprétation difficile. Ce recouvrement par des protéines d'hôte doit, au moins en partie, concourir à masquer le micro-organisme en facilitant sa fixation aux tissus, vraisemblablement préférentielle dans les sites inflammatoires ou les lésions. Quoi qu'il en soit, l'aptitude des Candida à fixer des protéines ou des glycoprotéines d'hôte est une caractéristique majeure, facilement mise en évidence (fig. 2 b). En ce qui concerne les bases moléculaires de cette fixation, il est remarquable de constater que les récepteurs pour le complément, la laminine et le fibrinogène présentent les mêmes masses moléculaires apparentes (68 000 et 60000 ) (1). Ces composants pariétaux portent donc très vraisemblablement des récepteurs multifonctionnels jouant un rôle essentiel dans les processus de colonisation et d'invasion.

\section{- La phagocytose et la candidicidie}

\section{- Les polynucléaires neutrophiles}

Les polynucléaires jouent un rôle essentiel dans les réactions de défense envers les Candida. Ainsi, une neutropénie inférieure à 500 éléments $/ \mathrm{mm}^{3}$ est considérée comme un facteur de risque candidosique majeur; les syndromes cliniques associés à des déficiences des fonctions des polynucléaires incluent des formes cutanéo-muqueuses chroniques de candidoses ou encore des formes systémiques. Chez le sujet sain, les différentes composantes de l'activité microbicide du polynucléaire sont actives sur les Candida, qu'il s'agisse du choc oxydatif, de la cathepsine D ou des défensines (7). Les mécanismes possibles de résistance de C. albicans sont la captation de la fraction C3 du complément qui inhiberait la fermeture de la vacuole de phagocytose et l'interaction du mannane avec le système myéloperoxydasique, induisant une réduction de $30 \%$ de la lyse des cellules ingérées.

\section{Les monocytes et les macrophages}

Aucun rôle direct de la lignée monocytaire dans l'élimination des Candida infectant l'organisme n'a pu être clairement démontré. Il est par contre fort probable que les mannoprotéines pariétales spontanément relarguées au cours des infections puissent interagir avec le récepteur pour le mannose des macrophages. Il a pu être mis en évidence d'autre part, que les patients souffrant de formes chroniques de candidoses présentaient un défaut de catabolisme du mannane, et que des macrophages provenant de ces sujets étaient susceptibles d'inhiber la prolifération lymphocytaire de sujets sains, vraisemblablement à la suite de la sécrétion de prostaglandines (2). Ces observations interfèrent sans doute peu ou prou avec le rôle essentiel du macrophage qui est celui de présenter l'antigène lors de l'induction de la réponse immune et sur lequel nous reviendrons brièvement ultérieurement.

\section{- La réponse anticorps}

Les anticorps n'ont qu'une action inhibitrice mineure sur la croissance des Candida in vitro et donc vraisemblablement pas de rôle protecteur direct au cours de l'infection. La nature des antigènes identifiés par les sérums de patients a par contre fait l'objet de nombreux travaux en vue d'améliorer la spécificité du diagnostic sérologique.

\section{- Anticorps anti-protéines}

Une protéine cytoplasmique majeure de $48 \mathrm{kDa}$ a été identifiée (14). Cette protéine, faiblement glycolysée, présentant une activité énolasique, serait localisée dans le système vacuolaire. Les anticorps dirigés contre cette protéine, ou des protéines de masses moléculaires apparentées (47 ou $45 \mathrm{kDa}$ ) ont été décrites par de nombreuses équipes comme étant synthétisés spécifiquement lors du développement pathogène de C. albicans. Il a pu être démontré, en outre, que l'antigène $48 \mathrm{kDa}$ circulait dans le sérum de patients, lors de processus invasifs sévères. L'existence d'une corrélation entre la présence d'anticorps anti-47 $\mathrm{kDa}$ et un état de protection des patients a été décrite sans jusqu'ici avoir reçu de confirmation (9). De même, la possibilité d'une sécrétion de l'antigène $47 \mathrm{kDa}$ au travers de la paroi fongique est encore discutée.

\section{. Anticorps anti-polysaccharides}

A l'inverse des glucanes, les mannanes pariétaux sont extrêmement antigéniques. C'est au sein des mannanes, dont la chimie commence à révéler la complexité (6) (fig. 2), que 
l'on trouve les déterminants antigéniques spécifiques de genre d'espèce et de sérotype ayant abouti à l'établissement d'une véritable classification des levures à l'aide de sérums polyclonaux monospécifiques. La signification diagnostique et pronostique des anticorps anti-mannanes est très controversée. Ces anticorps sont retrouvés dans le sérum de très nombreux sujets sains et augmentent parallèlement à la colonisation saprophytique, indépendamment de toute infection. Des études menées avec des anticorps polyclonaux ont cependant démontré qu'une variabilité antigénique de ces mannanes se manifestait en relation avec l'expression du pouvoir pathogène (11). Les études plus récentes avec les anticorps monoclonaux ont largement confirmé le caractère extrêmement variable, apparemment erratique de l'expression de surface des épitopes oligomannosidiques. Ces épitopes, qui peuvent être partagés par la copule polysaccharidique de nombreuses glycoprotéines cytoplasmiques ou pariétales, structurales ou enzymatiques, sont en outre retrouvés dans le sérum de patients atteints d'infections sévères (12).

\section{- L'immunomodulation}

De nombreuses observations ont prouvé que les mannanes de la paroi de $C$. albicans possédaient des propriétés immunomodulatrices (2). A cet égard, les études biocliniques conduites chez les patients atteints de formes sévères de candidoses sont particulièrement significatives. Il a été démontré qu'un facteur sérique existant dans le sérum de ces patients supprimait la réponse lymphocytaire de sujets sains vis-à-vis d'antigènes de $C$. albicans et que ce facteur disparaissait après guérison clinique de la candidose. Ce facteur a été identifié comme contenant du mannane; d'autres travaux ont par contre abouti à démontrer que le facteur inhibant la lymphoprolifération était représenté par des anticorps anti-Candida, ces deux types de résultats ne s'excluant d'ailleurs pas l'un l'autre. Ultérieurement, il a pu être démontré que les mannanes étaient susceptibles d'induire des populations de cellules $\mathrm{T}$ suppressives par l'intermédiaire de monocytes. Les mannanes présentent en outre des propriétés immunologiques peu communes : la capacité de se fixer aux cellules $\mathrm{T}$ et celle d'activer directement les cellules B (8). L'analyse fine des mécanismes mis en jeu dans de telles interactions relève de l'immunologie fondamentale. Les études conduites sur de nombreux modèles confirment que le terme d'immunomodulation est parfaitement bien adapté à la description de l'effet des mannanes puisqu'il dépend de l'animal, des doses et des modalités d'administration. Cependant, les résultats sont malheureusement d'interprétation difficile eu égard à la complexité des préparations antigéniques utilisées, à leur manque de standardisation, joint au manque de connaissances sur l'immunochimie des sucres.

\section{IV - Conclusions, perspectives, intérêt parasitologique du modèle}

Cette brève revue aboutit à dégager deux des originalités de ce modèle parasitologique. La première est le rôle prépon- dérant de la paroi, structure vivante en constante évolution, à l'interface des relations hôte-parasite. La seconde est l'importance physiopathologique d'une famille de composants pariétaux, les mannanes, qui interviennent dans chacune des étapes clefs de l'expression du pouvoir pathogène. Qu'il s'agisse de l'adhérence, de la pénétration, des interactions avec les composants spécifiques ou non spécifiques de l'immunité, les bases moléculaires des interactions de ces mannanes avec les composants cellulaires et humoraux de l'hôte sont essentielles à définir. Il est nécessaire en particulier de définir les relations entre structure et activité des multiples composants du répertoire oligosaccharidique de $C$. albicans. L'analyse immunochimique des sucres est un domaine difficile, en pleine expansion (y compris en parasitologie), qui contrairement à l'étude des protéines, échappe en grande partie aux méthodes de biologie moléculaire. Une des particularités des mannanes de $C$. albicans est de présenter des séquences d'oligomannosides, hautement conservées sur le plan phylogénétique, que l'on retrouve dans les stades précoces de la biosynthèse des glycoprotéines de nombreux protozoaires et métazoaires (fig. 2). La facilité avec laquelle il est possible de produire in vitro des quantités considérables d'oligosaccharides purifiés de levures pourrait être un facteur déterminant dans l'élucidation du rôle des structures oligomannosidiques des glycoprotéines dans les relations hôte-parasite.

De nombreuses techniques de biologie moléculaire sont appliquées depuis quelques années à l'étude de la pathogénicité des Candida. Ces études sont rendues extrêmement difficiles par la diploïdie du germe et l'absence de cycle sexué. La perspective la plus intéressante à l'heure actuelle concernant les bases de l'opportunisme de $C$. albicans est l'expression de phénotypes alternatifs (13), qui, par son caractère spontané, fournirait régulièrement au sein de la population cellulaire des individus présentant les caractères les plus adaptés à un changement de l'environnement.

\section{RÉFÉRENCES}

1. Bouchara J. P., Tronchin G., Annaix V., Robert R., Senet J. M. : Laminin receptors on Candida albicans germ tubes. Infect. Immun., 1989, 58, 48-54.

2. Domer J. : Candida cell wall mannan: a polysaccharide with diverse immunologic properties. Crit. Rev. Microbiol., 1989, 17, 33-51.

3. Dujardin L., Walbaum S. : Apport de la théorie des catastrophes à la description de la morphogénèse de Candida albicans. Physiologie Végétale, 1985, 23, 309-320.

4. Ghannoum M., Radwan S. : Candida adherence to epithelial cells. 1990, CRC Press Inc. (sous presse).

5. Gow N. A. R. : Biochemical and biophysical aspects of dimorphism in Candida albicans. In : Proceedings of the X ISHAM congress (J. M. Torres, ed.), J. R. Prous Science, barcelona, 1988, $73-77$.

6. Kobayashi H., Shibata N., Mitobe H., Ohkubo Y., Suzuki S. : Structural study of phosphomannan of yeast-form cells of Candida albicans $\mathbf{J}-1012$ strain with special reference to application of mild acetolysis. Archiv. Biochem. Biophys., 1989, 272, 364-375. 
7. Lehner R. : Antifungal mechanisms of leucocytes. Jap. J. Med. Mycol., 1988, 29, 1-6.

8. Mangeney M., Fischer A., Le Deist F., Latgé J. P., Durandy A. : Direct activation of human B lymphocytes by Candida albicans-derivated mannan antigen. Cell. Immunol., 1989, 122, 329-337.

9. Matthews R., Burnie J., Smith D., Clark I., Midgley J., Conolly M., Gazzard B. : Candida and AIDS: evidence for protective antibody. Lancet, 1988, i, 263-266.

10. Odds F. C. : Candida and candidosis. A review and bibliography (F. C. Odds Ed). Baillière and Tindall, London, 1988.

11. Poulain D., Hopwood V., Vernes A. : Antigenic variability of Candida albicans. CRC Crit. Rev. Microbiol., 1985, 12, 99-112.

12. Poulain D., Faille C., Michalski J. C., Camus D. : Anticorps monoclonaux anti-Candida albicans : de la biologie cellulaire aux marqueurs du comportement pathogène. Méd. Sci., 1990 (sous presse).

13. Slutsky B., Buffo J., Soll D. : High-frequency switching of colony morphology in Candida albicans. Science, 1985, 230, 666-669.

14. Strockbine N. A., Largen M. T., Zweibel S. M., Buckley H. : Identification and molecular weight characterization of antigens from Candida albicans that are recognized by human sera. Infect. Immun., 1984, 43, 715-721.

15. Tronchin G., Bouchara J. P., Robert R., Senet J. M. : Adherence of Candida albicans germ tubes to plastic : ultrastructural and molecular studies of fibrillar adhesins. Infect. Immun., 1989, 56, 1987-1993. 